\title{
Customizable electron beams from optically controlled laser plasma acceleration for $\gamma$-ray sources based on inverse Thomson scattering
}

\author{
S. Y. Kalmykov ${ }^{\mathrm{a}, *}$, X. Davoine ${ }^{\mathrm{b}}$, I. Ghebregziabher ${ }^{\mathrm{c}}$, B. A. Shadwick ${ }^{\mathrm{a}}$ \\ ${ }^{a}$ Department of Physics and Astronomy, University of Nebraska - Lincoln, Lincoln, NE 68588-0299, USA \\ ${ }^{b}$ CEA, DAM, DIF, Arpajon F-91297, France \\ ${ }^{c}$ Department of Mechanical and Nuclear Engineering, The Pennsylvania State University, University Park, PA 16802, USA
}

\begin{abstract}
Laser wakefield acceleration of electrons in the blowout regime can be controlled by tailoring the laser pulse phase and the plasma target. The $100 \mathrm{~nm}$-scale bandwidth and negative frequency chirp of the optical driver compensate for the nonlinear frequency red-shift imparted by wakefield excitation. This mitigates pulse self-steepening and suppresses continuous injection. The plasma channel suppresses diffraction of the pulse leading edge, further reducing self-steepening, making injection even quieter. Besides, the channel destabilizes the pulse tail confined within the accelerator cavity (the electron density "bubble"), causing oscillations in the bubble size. The resulting periodic injection generates background-free comb-like beams - sequences of synchronized, low phase-space volume bunches. Controlling the number of bunches, their energy, and energy spacing by varying the channel radius and the pulse length (as permitted by the large bandwidth) enables the design of a tunable, all-optical source of polychromatic, pulsed $\gamma$-rays using the mechanism of inverse Thomson scattering. Such source may radiate $\sim 10^{7}$ quasi-monochromatic $10 \mathrm{MeV}$ scale photons per shot into a microsteradian-scale observation angle. The photon energy is distributed among several distinct bands, each having sub-25\% energy spread dictated by the mrad-scale divergence of electron beam.
\end{abstract}

Keywords:

Laser wakefield acceleration, blowout, plasma channel, electron trapping, negative chirp, inverse Thomson scattering PACS: 41.75.Jv, 41.85.Ct, 42.65.Jx, 42.65.Wi, 52.35.Mw, 52.38.Hb, 52.38.Kd, 52.38.Ph

\section{Introduction}

Electron beams from laser-plasma accelerators (LPAs) $[1,2]_{25}$ are the key element of all-optical X- and $\gamma$-ray sources based ${ }_{26}$ on the mechanism of inverse Thomson scattering (ITS) [3-12]. 27 The electron beam phase space structure imprints onto the ITS 28 $\gamma$-ray spectrum. This structure is highly susceptible to the details of the drive laser pulse evolution. The latter may be de- ${ }_{30}$ liberately controlled through manipulations of the drive pulse ${ }_{31}$ phase, thus affording considerable freedom in electron beam shaping [13-16]. Using the large-bandwidth negatively chirped ${ }_{33}$ pulse (NCP) as a driver helps suppress continuous injection and ${ }_{34}$ accumulation of a massive low-energy tail $[15,16]$. Modern ${ }_{35}$ laser technology offers $100 \mathrm{~nm}$-scale bandwidth [17] and the ${ }_{36}$ expectation of the $\mathrm{kHz}$-repetition-rate, Joule-scale pulses [18] ${ }_{37}$ necessary for the practical realization of the concept [19].

This report describes a path to controlled production of cus- ${ }_{39}$ tomizable comb-like electron beams - synchronized sequences ${ }_{40}$ of fs-length, multi-kA quasi-monoenergetic (QME) bunches. ${ }_{41}$ These are hard to obtain with conventional accelerators $\left[20-{ }_{42}\right.$ 23 , yet emerge naturally in LPAs [24, 25]. Comb-like beams, ${ }_{43}$ associated with multiple injections into the first bucket [26-28], ${ }_{44}$ or into consecutive buckets of the laser wake [29], have been ${ }_{45}$

${ }^{*}$ Corresponding author. Tel.: +1 402261 3815, fax: +1 4024726148 .

Email address: skalmykov2@unl .edu (S. Y. Kalmykov)

(1)
the dynamics responsible for multiple injection, and produce the energy combs in a controlled fashion, we propose propagating the NCP in a plasma channel $[16,30]$, while carrying out acceleration in the blowout regime [31]. The preformed channel [32] is generally known to stabilize the acceleration process $[33,34]$. In the blowout regime, however, the situation is more subtle. The radiation pressure of the strongly overcritical pulse, $P \gg P_{\mathrm{cr}}=16.2\left(\omega_{0} / \omega_{p e}\right)^{2} \mathrm{GW}$, with a length close to a Langmuir period, $\omega_{p e} \tau_{L} \sim 2 \pi$, creates complete electron cavitation, leaving the background ions unperturbed [35-37]. Here, $\omega_{p e}=\left(4 \pi e^{2} n_{0} / m_{e}\right)^{1 / 2} \ll \omega_{0}$ is the Langmuir electron frequency, $\omega_{0}$ is the pulse carrier frequency, $n_{0}$ is the background electron density, $-|e|$ and $m_{e}$ are the electron charge and mass. The resulting "bubble" of electron density self-consistently guides the pulse over many Rayleigh lengths [31], with a minimal assistance from the preformed channel. Two other aspects, however, become essential. First, the channel suppresses diffraction of the NCP leading edge, thus further reducing the pulse selfsteepening. Secondly, and most importantly, the external channel may destabilize the tail of the pulse confined within the bubble, bringing about oscillations in the bubble size (the accordion effect), producing periodic injection and formation of a train of fs-length electron bunches with a micron-scale emittance and controlled energy spacing [30]. With the number of bunches controlled by changing either the channel radius or the pulse 
length, the customizable, comb-like beams are ideal drivers of tunable, multi-color ITS $\gamma$-ray sources.

The paper is organized as follows. Section 2 describes the computational approach and defines parameters of the case studies. Section 3 demonstrates the effect of negative chirp of the driver on the electron acceleration process. Section 4 demonstrates generation of clean comb-like beams in a channel, outlines the physical phenomena underlying the accordion effect, and points out various control options for the electron spectra. Section 5 focuses on generation of polychromatic ITS $\gamma$-rays with the energy reaching $12.5 \mathrm{MeV}$. Section 6 summarizes the results and points out directions of future work.

\section{Simulation framework}

Reduced and full 3D PIC simulations elucidate the physical processes essential for electron beam shaping. Mitigation of adverse optical phenomena through shaping the laser pulse phase is explored in fast quasistatic simulations using the fully relativistic, cylindrically symmetric, optical cycle-averaged code WAKE $[35,36]$. Owing to the quasistatic description of plasma, WAKE separates the details of bubble dynamics brought about by the evolution of the optical driver from the effects brought about by the collective fields of trapped electrons, i.e. from effects due to beam loading $[15,38]$. The complex envelope of the laser vector potential is computed using an extended paraxial solver. It preserves group velocity dispersion in the presence of large frequency shifts and accurately calculates laser pulse absorption due to wake excitation [39, 40]. To correctly capture all physics relevant to pulse propagation and evolution of ${ }^{104}$ the bubble, we use the grid $\Delta \xi \approx \Delta r / 3 \approx \lambda_{0} / 13 \approx 63 \mathrm{~nm}$ and $^{105}$ time step $\omega_{0} \Delta t \approx 1.325$, taking 30 macroparticles per radial ${ }^{106}$ cell. Here, $\xi=z-c t, r^{2}=x^{2}+y^{2}$, and $\omega_{0}=2 \pi c / \lambda_{0}$. Figures $1^{107}$ and 2 show the results of WAKE simulations.

Self-consistent simulations of electron phase space dynamics ${ }^{109}$ are carried out with the quasi-cylindrical PIC code CALDERCirc [41]. The numerical Cherenkov-free electromagnetic ${ }_{110}$ solver in CALDER-Circ [42], in combination with the thirdorder macroparticles, fine grid, $\Delta z \approx \Delta r / 16 \approx \lambda_{0} / 50 \approx 16 \mathrm{~nm}$, small time step, $\omega_{0} \Delta t=0.1244$, and 20 macroparticles per cell, ${ }^{111}$ helps maintain low sampling noise, negligibly low numerical ${ }^{112}$ dispersion, and avoids numerical emittance dilution. Figures ${ }^{113}$ 3-6 show the results of CALDER-Circ simulations.

The linearly polarized, $70 \mathrm{TW}$ laser pulse with the carrier wavelength $\lambda_{0}=0.805 \mu \mathrm{m}$ is focused at the plasma edge, ${ }_{115}$ $z=0$, and propagates in the positive $z$ direction. The initial complex amplitude of the vector potential, normalized to ${ }_{116}$ $m_{e} c^{2} /|e|$, is $\tilde{a}(z=0)=a_{0} \exp \left[-\left(r / r_{0}\right)^{2}-2 \ln 2\left(t / \tau_{L}\right)^{2}+i \varphi(t)\right]_{, 117}$ where $a_{0}=3.27$ and $r_{0}=13.6 \mu \mathrm{m}$. The rate of phase vari-118 ation defines the instantaneous frequency, $\omega(t)=-\mathrm{d} \varphi / \mathrm{d} t={ }_{119}$ $\omega_{0}-(4 \ln 2)\left(\kappa / \tau_{L}\right)^{2} t$, where $\omega_{0}=2 \pi c / \lambda_{0}$. If $\kappa \neq 0$, the higher ${ }_{120}$ frequencies are advanced in time, and the pulse is negatively $y_{121}$ chirped. Throughout the paper (except for the discussion of Fig. 6 ), we consider the pulse with a full width at half-maximum ${ }^{122}$ $\tau_{L}=30 \mathrm{fs}$, which is either transform-limited (TLP, $\kappa=0$ ) or $^{123}$ negatively chirped, $\kappa=2.432$. In the latter case, the NCP band-124 width is equivalent to a transform-limited duration $\tau_{L 0}=5 \mathrm{fs}, 125$

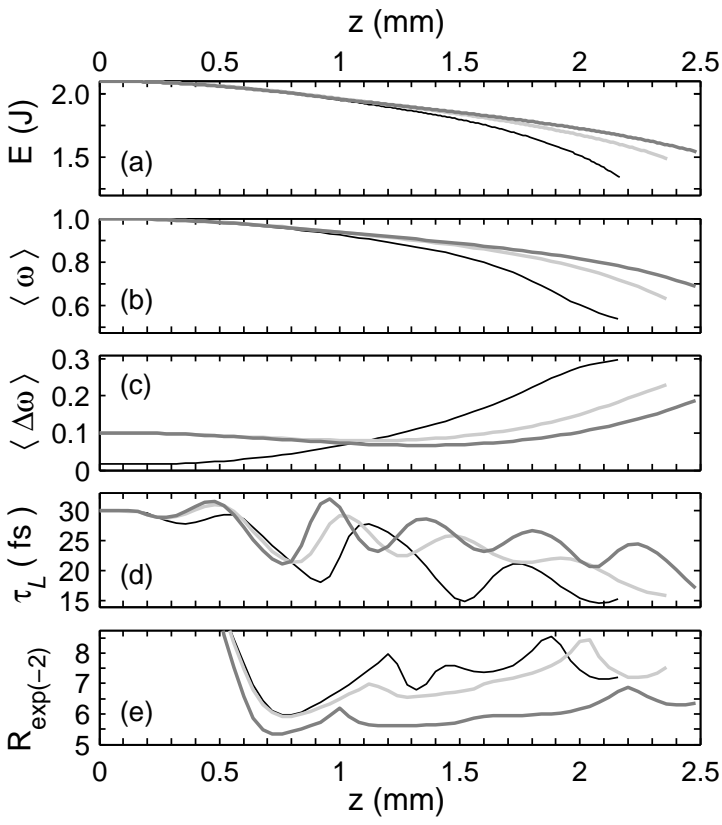

Figure 1: Evolution of (a) laser pulse energy, (b) mean frequency (in units $\omega_{0}$ ), (c) RMS bandwidth (in units $\omega_{0}$ ), (d) pulse length computed from the $\zeta$ variance of energy density on axis, (e) local radius, in microns [taken at exp $(-2)$ of the peak intensity in the highest-intensity cross-section of the pulse]. Blackthe Reference case; light gray - the NCP in the uniform plasma; dark gray - the NCP in the channel. The curves terminate as soon as electrons reach dephasing. The negative chirp, in combination with the channel, reduces depletion, frequency red-shift, spectral broadening, and contraction of the pulse.

which yields full width at half-maximum in spectral intensity $\Delta \lambda=\sqrt{2 \ln 2}\left(\lambda_{0}^{2} / \pi\right)\left(c \tau_{L 0}\right)^{-1} \approx 160 \mathrm{~nm}$.

The plasma begins at $z=0$ with a $0.5 \mathrm{~mm}$ linear ramp, followed by a longitudinally flat section with a density on axis $n_{0}=6.5 \times 10^{18} \mathrm{~cm}^{-3}$. We explore new directions in electron beam shaping by propagating the pulse in a leaky channel:

$$
n_{e}(r)= \begin{cases}n_{0}\left(1+r^{2} / r_{\mathrm{ch}}^{2}\right) & \text { for } r \leq r_{\mathrm{ch}}, \\ 2 n_{0}\left(2-r / r_{\mathrm{ch}}\right) & \text { for } r_{\mathrm{ch}}<r \leq 2 r_{\mathrm{ch}}, \\ 0 & \text { for } r>2 r_{\mathrm{ch}} .\end{cases}
$$

We present a comparative study of three cases: the TLP in the uniform plasma (the Reference case); the NCP in a uniform plasma, $r_{\mathrm{ch}}=\infty$; the NCP in a channel matched to the selfguided spot size, $r_{\mathrm{ch}}=37.5 \mu \mathrm{m}$.

\section{Negative chirp: A remedy for nonlinear pulse distortion}

The changes in pulse evolution brought about by the frequency chirp are tracked using the full vector potential, $\tilde{a}=$ $a(r, z, \zeta) \mathrm{e}^{-i \omega_{0} \zeta}$, where $\zeta=\xi / c$, and $a(r, z, \zeta)$ is the complex envelope from WAKE simulations. Figures 1(b) and 1(c) show the radially integrated mean frequency $\langle\omega(z)\rangle=$ $A^{-1} \int_{0}^{\infty} r \mathrm{~d} r \int_{0}^{\infty} \omega|\tilde{a}(r, z, \omega)|^{2} \mathrm{~d} \omega$ and frequency variance $\langle\Delta \omega(z)\rangle=$ $\left(A^{-1} \int_{0}^{\infty} r \mathrm{~d} r \int_{0}^{\infty}(\omega-\langle\omega\rangle)^{2}|\tilde{a}(r, z, \omega)|^{2} \mathrm{~d} \omega\right)^{1 / 2}$, where $\tilde{a}(r, z, \omega)=$ $\int_{-\infty}^{+\infty} \tilde{a}(r, z, \zeta) \mathrm{e}^{-i \omega \zeta} \mathrm{d} \zeta$, and $A(z)=\int_{0}^{\infty} r \mathrm{~d} r \int_{0}^{\infty}|\tilde{a}(r, z, \omega)|^{2} \mathrm{~d} \omega$. Figure $1(\mathrm{~d})$ shows the pulse length computed from the $\zeta$-variance of intensity on axis, $\tau_{L}(z)=\left(8 \ln 2 B^{-1} \int_{-\infty}^{\infty}(\zeta-\langle\tau\rangle)^{2}|a(0, z, \zeta)|^{2} \mathrm{~d} \zeta\right)^{1 / 2}$. 

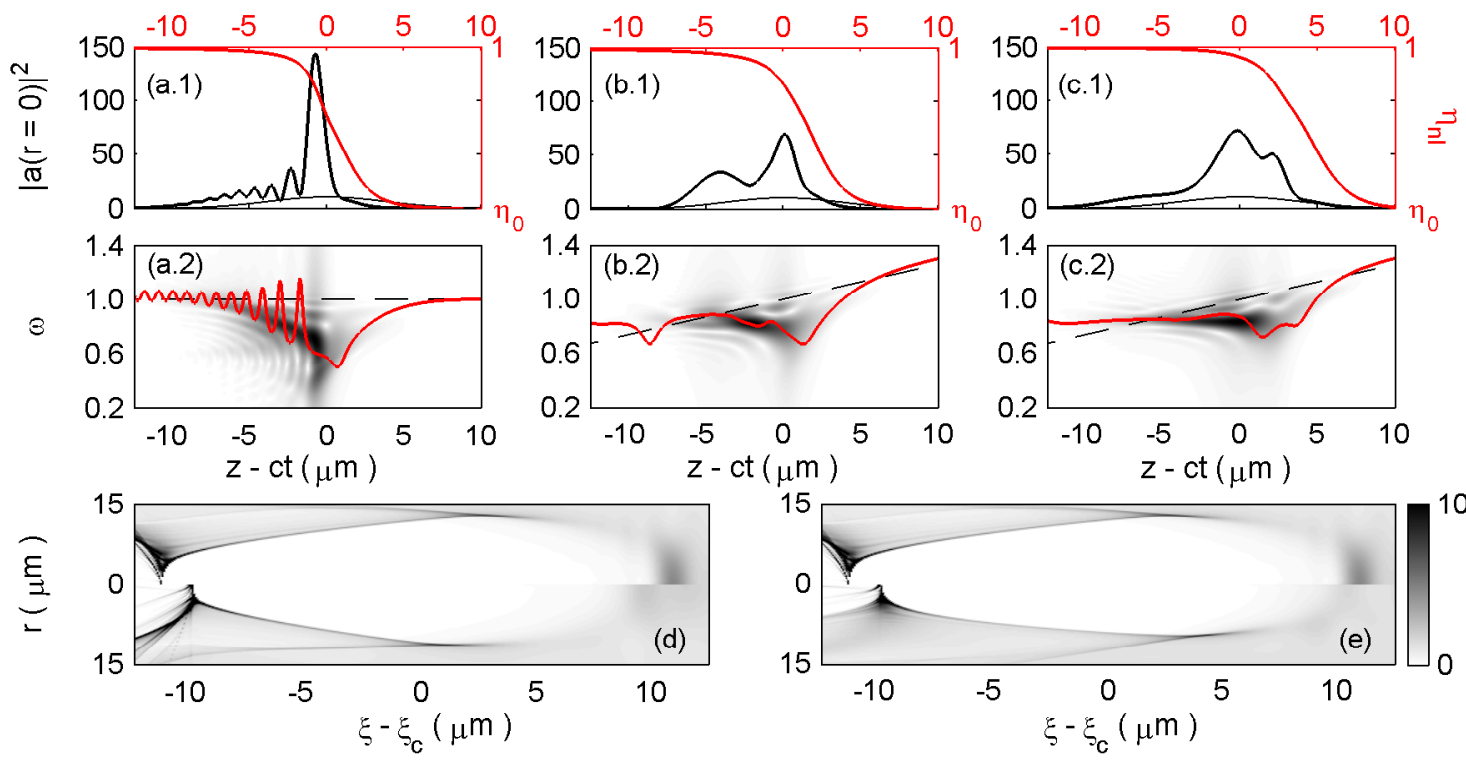

Figure 2: Mitigating self-compression of the pulse and expansion of the bubble with the negative chirp. (a) The Reference case. (b) The NCP in a uniform plasma and (c) in a channel. All quantities are shown at $z=1.72 \mathrm{~mm}$, long before electron dephasing. Pulse propagates from left to right. (a.1)-(c.1) Axial lineouts of $|a|^{2}$ (left axes; thin curve is the initial profile) and of the nonlinear refractive index [right axes, red (gray) curve]; $\eta_{0}=0.99812$ is the linear refractive index of the plasma. (a.2)-(c.2) Absolute value of the Wigner transform (grayscale) and instantaneous frequency (in units $\omega_{0}$ ) extracted from the envelope phase. Dashed lines show the instantaneous frequency of the incident pulse. (d), (e) Quasistatic cavity of electron density. The density is normalized to $n_{0}$; $\xi=\xi_{c}$ is the bubble centerplane, where the longitudinal electric field vanishes. Top halves correspond to the Reference case. Bottom halves in (d) and (e) correspond to the NCP in the uniform plasma and in a channel, respectively. In the Reference case, the pulse acquires robust positive chirp. Its leading edge red-shifts by an almost half the carrier frequency and self-compresses into a cycle-long optical shock. Conversely, plasma response merely un-chirps the NCP, reducing pulse compression and bubble expansion.

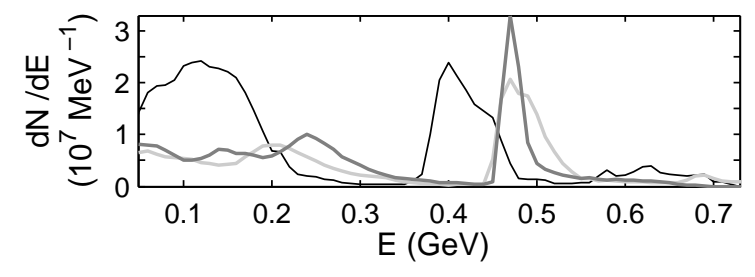

Figure 3: Electron energy spectra corresponding to the cases displayed in Fig. 2. Thin black curve: the Reference case; light (dark) gray: the NCP in a uniform plasma (in a channel). Chirp and channel give the QME feature a 20\% energy boost, while considerably reducing its bandwidth and suppressing the tail.
Here, $\langle\tau(z)\rangle=B^{-1} \int_{-\infty}^{\infty} \zeta|a(0, z, \zeta)|^{2} \mathrm{~d} \zeta$ is the position of the pulse centroid, and $B(z)=\int_{-\infty}^{\infty}|a(0, z, \zeta)|^{2} \mathrm{~d} \zeta$.

Figure 2 links longitudinal distortion of the pulse to the local frequency shift. The shift is extracted from the pulse envelope phase on axis, $\tilde{a}(0, \zeta)=|a| \mathrm{e}^{-i \omega_{0} \zeta+i \phi}$, using two indepen- ${ }_{143}$ dent methods [39]. Firstly, the Wigner transform $\mathcal{W}(\zeta, \omega)={ }_{144}$ $(2 \pi)^{-1} \int_{-\infty}^{+\infty} \tilde{a}^{*}\left(\zeta+\zeta^{\prime} / 2\right) \tilde{a}\left(\zeta-\zeta^{\prime} / 2\right) \exp \left(i \omega \zeta^{\prime}\right) \mathrm{d} \zeta^{\prime}$ yields the distri- ${ }_{145}$ bution of "photon density" in the "photon phase space" $(\zeta, \omega)_{\cdot 146}$ Secondly, we calculate the instantaneous frequency using the ${ }_{147}$ rate of the envelope phase change, $\omega(\zeta)=\omega_{0}-\mathrm{d} \phi / \mathrm{d} t={ }_{148}$ $\omega_{0}-\partial \phi / \partial \zeta$. Mean frequency, RMS bandwidth, pulse duration, ${ }_{149}$ and photon density are experimentally measurable markers of ${ }_{150}$ nonlinear optical processes. They help identify the regimes of ${ }_{151}$ pulse propagation and wakefield excitation [43-45].

The acceleration process in the Reference case is ruined long ${ }_{153}$ before electron dephasing. Figures 1(a)-1(d) show that, by de-154 phasing, the energy, mean frequency, and the RMS duration ${ }_{155}$

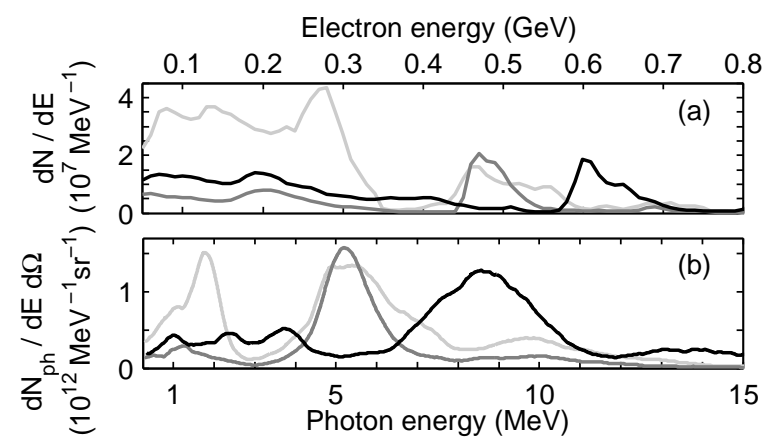

Figure 4: Monochromatizing the ITS $\gamma$-rays. (a) Electron energy spectra. Light gray: the Reference case, $z=2.16 \mathrm{~mm}$ (dephasing). Dark gray (black): $30 \mathrm{fs}$ NCP in a uniform plasma, $z=1.72 \mathrm{~mm}(2.16 \mathrm{~mm})$. (b) The ITS $\gamma$-ray flux on axis, in the direction of electron beam propagation.

of the TLP drop nearly by half, while the RMS frequency bandwidth increases by an order of magnitude. Figures 2(a.1) and 2(a.2) demonstrate that a co-moving negative gradient in the nonlinear index red-shifts the TLP leading edge by a half$\omega_{0}$, producing a cycle-long optical shock. Snow-plowing by the shock compresses electron fluid in the front of the bubble [cf. Figs. 2(d) and 2(e) top], increasing charge separation, imparting relativistic (negative) longitudinal momentum to the sheath electrons. The resulting delay in their return to the axis elongates the bubble [15]. The associated continuous injection builds up a massive energy tail, seen in Fig. 3, black. By dephasing, over $80 \%$ of the accelerated charge belongs to the tail; see Fig. 4(a), light gray. Plasma channel alone is unable to im- 


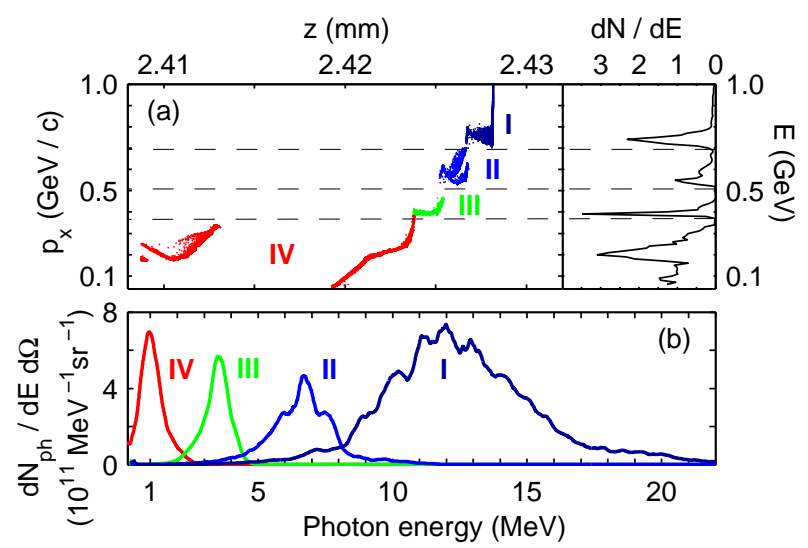

Figure 5: Generating polychromatic ITS $\gamma$-rays with a background-free, comblike electron beam. (a) Longitudinal phase space and energy spectrum (inset) of ${ }^{204}$ the comb-like beam accelerated with the NCP through dephasing in a channel, ${ }^{205}$ $z=2.48 \mathrm{~mm}$. Electrons propagate to the right. The four energy intervals corre-206 spond to individual components of the comb. The beam statistics are presented $\mathrm{d}_{207}$ in table 1. (b) The ITS $\gamma$-ray flux from individual electron bunches.

the$$
\text { ene }
$$

prove the situation [16].

The negative frequency chirp avoids this unfavorable sce- ${ }^{211}$ nario, and allows acceleration through dephasing without sac $^{212}$ rificing beam quality. Figures 1(a)-1(d) show that, in contrast ${ }^{213}$ to the Reference case, the NCP depletes and red-shifts in fre-- ${ }^{214}$ quency by less than one-third. Temporal advancement of high $^{215}$ frequencies compensates for the nonlinear red-shift. From Figs. ${ }^{216}$ 2(b) and 2(c), at the point of TLP transformation into an optical ${ }^{217}$ shock, the NCP has almost flat frequency profile, and is as yet ${ }^{218}$ uncompressed. This avoids the snow-plow effect and reduces ${ }^{219}$ expansion of the bubble [cf. Figs. 2(d) and 2(e)]. The monoenergetic peak thus dominates electron spectra through dephasing, ${ }_{220}$ while receiving $30 \%$ boost in energy [Fig. 4(a)].

\section{Generation of comb-like beams in plasma channel}

By altering the kinetics of self-injection, the channel allows ${ }^{224}$ further customization of electron beam. The channel upsets the $\mathrm{e}^{225}$ balance between the radiation pressure of the pulse tail confined inside the bubble and the radial restoring force due to the charge separation. As a result, the tail flaps, driving the bubble boundaries sidewards, changing the bubble size [30], yielding four localized electron injection events. Electron beam thus splits into a sequence of distinct quasi-monochromatic bunches shown in Fig. 5(a). Statistics of these bunches are presented in Table 1. Periodic focusing of the pulse tail replicates itself in regular oscillations of the pulse RMS length on axis; see Fig. 1(d), dark gray. Conversely, evolution of the pulse spot size - the pulse radius in the highest-intensity cross-section [at $\exp (-2)$ of the peak intensity] - is insensitive to the transverse inhomogeneity of the plasma, except for the $15 \%$ reduction in the spot size; see Fig. 1(e). Thus, contrary to the earlier conjectures [24, 25], the channel stabilizes the most intense segment of the pulse, preventing its undamped periodic focusing.

By suppressing diffraction of the NCP leading edge, the channel further reduces pulse self-steepening, thereby delay- ing dephasing of the leading bunch [labeled I in Fig. 5(a)]. The latter receives additional $17 \%$ energy boost against the uniform plasma case, reaching $770 \mathrm{MeV}$; compare the entries Mono-I and $\mathbf{I}$ in Table 1. By dephasing, the four-component beam absorbs $15.7 \%$ of the NCP energy, while having 70-120 kA current of fs-length individual bunches.

The accordion effect preserves electrons in the second bucket [a trailing component of the lowest-energy bunch (IV) in Fig. 5(a)]. They are injected early and dephase early, staying below $300 \mathrm{MeV}$, contributing to the lowest-energy signal. As injection in the second bucket never resumes, this group remains quasimonoenergetic; entry IV-2 in Table 1 contains their statistics.

Reducing the amount of radiation confined inside the bubble eliminates the accordion effect. Reducing the NCP length by one-third while preserving the power and bandwidth $(\kappa=$ 1.968 ), and also increasing the channel radius by $40 \%$, reduces the energy comb to a single high-flux component absorbing $16.7 \%$ of the NCP energy [beam Mono-II in Fig. 6(a)]. Yet the energy tail remains as weak as in the uniform plasma. Changing the channel radius [30] and/or the NCP length thus effectively controls the output of the acceleration process and the electron beam structure, from comb-like to quasi-monoenergetic.

Last but not least, in all cases examined here, the QME electron bunches are accelerated through dephasing with their normalized transverse emittance varying in the third digit only, thanks to the numerical Cherenkov-free electromagnetic solver of the CALDER-Circ code [42]. Thus any degradation of the ITS $\gamma$-ray signal observed in the simulations can be attributed to physical causes, such as mrad-scale electron beam divergence or accumulation of the modest low-energy tail.

\section{Polychromatic $\gamma$-rays from inverse Thomson scattering}

We extract $N_{\mathrm{b}}$ macroparticles from the first and second buckets of the laser wake, sample the six-dimensional phase space of electron beam with their coordinates and momenta, and then propagate these electrons in a free space by solving the relativistic equations of motion [46]. In the absence of the laser field,

\begin{tabular}{lrrrrrrc}
\hline Units & $\begin{array}{r}\langle E\rangle \\
\mathrm{MeV}\end{array}$ & $\begin{array}{r}\sigma_{E} \\
\mathrm{MeV}\end{array}$ & $\begin{array}{r}\sigma_{\alpha} \\
\mathrm{mrad}\end{array}$ & $\begin{array}{r}\varepsilon_{\perp}^{N} \\
\mu \mathrm{m}\end{array}$ & $\begin{array}{r}\tau_{b} \\
\mathrm{fs}\end{array}$ & $\begin{array}{r}Q \\
\mathrm{pC}\end{array}$ & $\begin{array}{c}N_{\mathrm{ph}} \\
10^{7}\end{array}$ \\
\hline Mono-I & 660 & 72 & 1.9 & 0.57 & 2.25 & 228 & 0.96 \\
Mono-II & 583 & 32 & 2.6 & 0.84 & 4.35 & 400 & 1.53 \\
I & 770 & 56 & 2.2 & 1.27 & 1.45 & 175 & 0.62 \\
II & 582 & 40 & 3.4 & 1.43 & 1.15 & 95 & 0.27 \\
III & 405 & 15 & 3.2 & 0.75 & 1.50 & 105 & 0.34 \\
IV-1 & 225 & 32 & 4.9 & 0.72 & 2.75 & 302 & 0.91 \\
IV-2 & 242 & 48 & 9.0 & 2.73 & 3.00 & 121 & 0.20 \\
\hline
\end{tabular}

Table 1: Statistics of QME electron beams at dephasing. Beams are labeled according to their markers in Figs. 5(a) and 6(a). $\langle E\rangle$ is the mean energy; $\sigma_{E}$ is the dispersion of energy; $\sigma_{\alpha}$ is the RMS divergence; $\varepsilon_{\perp}^{N}$ is the RMS normalized transverse emittance [30]; $\tau_{b}$ is the RMS bunch duration; and $Q$ is the charge. Electrons making up the beams IV-1 and IV-2 are accelerated in the first and second bucket, respectively. $N_{\mathrm{ph}}$ is the estimated number of photons in the observation solid angle $\Delta \Omega=\pi\left\langle\gamma_{e}\right\rangle^{-2}$, which corresponds to the quasimonochromatic $\gamma$-ray signals in Figs. 5(b) and 6(b). 


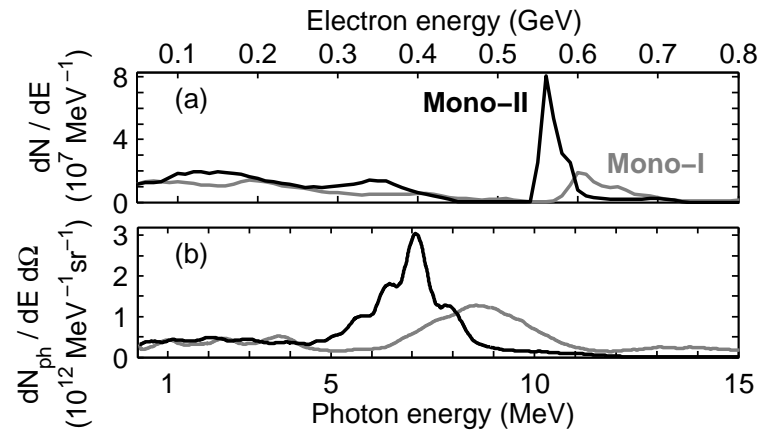

Figure 6: Generating quasi-monochromatic ITS $\gamma$-rays using QME electrons 271 from a channel or a uniform plasma. Gray: $30 \mathrm{fs}, 70$ TW NCP in the uniform ${ }_{272}$ plasma. Black: $20 \mathrm{fs}, 70 \mathrm{TW}$ NCP in a wide channel, $r_{\mathrm{ch}}=54.5 \mu \mathrm{m}$. (a) Electron energy spectra at dephasing, $z=2.16 \mathrm{~mm}$. Beam statistics are given in $\mathrm{Ta}^{273}$ ble 1. (b) The ITS $\gamma$-ray spectra. The beam Mono-I produces $9.6 \times 10^{6}$ photons ${ }^{274}$ in the observation angle $\Delta \Omega=\pi\left\langle\gamma_{e}\right\rangle^{-2}$, with the average energy $\left\langle E_{\mathrm{ph}}\right\rangle=8.6_{275}$ $\mathrm{MeV}$ and $16 \%$ RMS energy spread. The beam Mono-II produces $1.53 \times 10^{7}{ }_{276}$ photons, with $\left\langle E_{\mathrm{ph}}\right\rangle=7 \mathrm{MeV}$ and $18 \%$ spread.

their trajectories are ballistic. Since the beams considered here
are relativistic and low-density, $n_{c}\left\langle\gamma_{c}\right\rangle^{-3} \ll 10^{16} \mathrm{~cm}^{-3}$, space $^{280}$ are relativistic and low-density, $n_{e}\left\langle\gamma_{e}\right\rangle^{-3} \ll 10^{16} \mathrm{~cm}^{-3}$, space
charge forces are neglected [47, 48]. Radiation damping is also
281 neglected, as the energy emitted by an electron passing through ${ }^{282}$ the interaction laser pulse (ILP) is small compared to the en-283 ergy of the electron. The electron beam collides head-on with ${ }^{284}$ the linearly polarized ILP, which is specified analytically using ${ }^{285}$ the paraxial approximation. The ILP has a $0.8 \mu \mathrm{m}$ wavelength ${ }^{286}$ (photon energy $1.5 \mathrm{eV}$ ), 250 fs duration, and $20 \mu \mathrm{m}$ focal spot $^{287}$ size. Timing between the electron beam and the ILP is chosen ${ }^{288}$ so that the beam and the peak of the ILP intensity arrive at the ${ }^{289}$ ILP focal plane simultaneously. As the ILP is shorter than 5\% $\%^{290}$ of its Rayleigh length and the electron beam spot size is in the ${ }^{291}$ sub-micron range, the interaction occurs in an almost planar- ${ }^{292}$ wave geometry. To avoid broadening the ITS spectra $[49,50]]^{293}$ a linear interaction regime is chosen, with the ILP normalized vector potential $a_{\mathrm{int}}=0.1$. Once the orbits of individual elec-294 trons are obtained, the mean energy density radiated per unit frequency $\omega$ and solid angle $\Omega$ per electron [51], after averag ${ }_{2}^{295}$ ing over the ensemble, is given by

$$
\frac{d^{2} I_{e}}{d \omega d \Omega}=\left.\frac{A}{\sum_{i=1}^{N_{\mathrm{b}}} w_{i}} \sum_{i=1}^{N_{\mathrm{b}}} w_{i}\left|\int_{-\infty}^{\infty} \mathbf{n} \times\left(\mathbf{n} \times \boldsymbol{\beta}_{i}\right) \mathrm{e}^{i \omega\left(t-\mathbf{n} \cdot \mathbf{r}_{i}(t) / c\right)} d t\right|^{2}\right|_{300} ^{298}
$$

where $A=e^{2} \omega^{2} /\left(4 \pi^{2} c\right), w_{i}$ is the macroparticle weight, $\mathbf{n}$ is 302 the unit observation vector (pointing in the direction of electron 303 beam propagation), and $\mathbf{r}_{i}$ and $\boldsymbol{\beta}_{i}=\mathbf{v}_{i} / c$ are the radius vec-304 tor and normalized velocity of the test electron, respectively.305 Then, the net energy radiated by the beam with a charge $Q_{306}$ is $d^{2} I_{\mathrm{tot}} / d \omega d \Omega=(Q /|e|) d^{2} I_{e} / d \omega d \Omega$. The ITS $\gamma$-ray flux dis-307 played in Figs. 4(b)-6(b) is shown on axis, in the direction of ${ }_{308}$ electron beam propagation, and in the ILP polarization plane. 309

Damping the electron energy tail monochromatizes the ITS $_{310}$ signal. Figure 4(a) (dark gray) shows that the highest electron ${ }_{311}$ energy accessible in the Reference case $(\approx 460 \mathrm{MeV})$ can be ${ }_{312}$ reached with the NCP in a $25 \%$ shorter uniform plasma, with ${ }_{313}$ the charge in the tail reduced by a factor 6 . This clean electron ${ }_{314}$ beam radiates (estimated) $9.8 \times 10^{6}$ quasi-monochromatic photons $\left(\left\langle E_{\mathrm{ph}}\right\rangle=5.3 \mathrm{MeV}, \sigma_{E}=0.8 \mathrm{MeV}\right)$ in the observation solid angle $\Delta \Omega=\pi\left\langle\gamma_{e}\right\rangle^{-2} \approx 3.05 \times 10^{-6}$ sr. This ITS signal, shown in Fig. 4(b) (dark gray), lacks the background almost entirely, in striking contrast to the $100 \%$ background level in the Reference case. Further acceleration through dephasing increases electron energy by $30 \%$, while doubling the charge in the tail. The beam extracted at dephasing [Mono-I in Fig. 6(a)] radiates $9.6 \times 10^{6}$ photons in the observation angle $\Delta \Omega \approx 1.9 \times 10^{-6}$ sr. The ITS signal with $\left\langle E_{\mathrm{ph}}\right\rangle=8.6 \mathrm{MeV}$ and $\sigma_{E}=1.375 \mathrm{MeV}$ [Fig. 6(b), gray] resides on top of the continuous background with the flux below a quarter of the peak signal.

QME beam from the channel - Mono-II in Fig. 6(a) - has the same low-energy background as Mono-I, yet the flux so high as to double the $\gamma$-ray flux [cf. Fig. 6(b)] and increase the number of photons in the observation angle by $60 \%$ against the uniform plasma case. The estimated total energy radiated into the observation angle reaches $0.017 \mathrm{~mJ}$, which is an order-of-magnitude igher than yet achieved experimentally $[7,11]$.

The comb-like beam from the channel, characterized in Fig. 5 (a), lacks a tail almost entirely. It radiates $(2-9) \times 10^{6}$ photons into four distinct $\gamma$-ray energy bands; see Fig. 5(b) and Table 1. Milliradian-scale divergence of electron bunches imparts up to $25 \%$ RMS energy spread to the individual $\gamma$-ray beamlets. Post-manipulations using highly chromatic electron optics [53, 54] may improve electron beam collimation and slice energy spread, approaching a sub-percent $\gamma$-ray bandwidth demanded by nuclear photonics applications [52].

Demonstrated customization of the all-optical ITS $\gamma$-ray source is enabled exclusively by the $100 \mathrm{~nm}$-scale bandwidth of the LPA drive pulse, permitting manipulations of electron beam phase space in very wide limits. This is further evidence that new broadband pulse amplification techniques [18] are critically important for the design of future compact $\gamma$-ray sources.

\section{Summary and outlook}

Control of relativistic optical processes in an LPA permits beam quality control in the highly dispersive dense plasmas as well as generation of unconventional electron beams inaccessible with standard acceleration techniques. Looking ahead to the arrival of PW-class laser systems delivering sub-2-cycle pulses [18], we propose using their ultrahigh bandwidth to modify the electron beam phase space structure. First, manipulations of the drive pulse phase (negative chirp) improve the monochromaticity and boost the energy of $\mathrm{GeV}$-scale electrons, while preserving mm-scale size of the plasma and Joule-scale energy of the pulse. Secondly, propagating a negatively chirped pulse in a plasma channel destabilizes the accelerating bucket, causing oscillations in its size. This accordion effect causes periodic self-injection of ambient electrons and their subsequent acceleration with a low energy spread. The channel radius and the laser pulse length are important parameters, controlling the number of spectral components in the comb-like beam.

The comb of synchronized (on a fs-scale), virtually background-free $100 \mathrm{kA}$-current electron bunches is an asset for the design of compact radiation sources. Our first-principles 
simulations show that inverse Thomson scattering of an opti-378 cal beam off a comb-like electron beam yields multi-color, fs- ${ }^{379}$ length pulses of $\gamma$-rays. All-optical manipulation of the elec- ${ }^{380}$ tron beam phase space enables fine tuning parameters of these ${ }_{382}$ pulses, to the benefit of applications. The natural mutual syn-38з chronization of fs-length electron bunches and $\gamma$-ray flashes ${ }^{384}$ may be an asset to nuclear pump-probe experiments.

Constructing a piece-wise negative chirp by incoher- ${ }_{-387}^{386}$ ently stacking collinearly propagating, conventional narrow-388 bandwidth pulses of different colors promises additional de--389 grees of freedom in beam quality control [16]. Generat ${ }^{390}$ ing comb-like beams with these stacked drivers and optimiz- 392 ing them for the design of ITS-based radiation sources, using 393 application-specific metrics, is the focus of our future work.

\section{Acknowledgements}

The work of SYK and BAS was supported by the US DOE ${ }_{400}^{399}$ Grant DE-SC0008382 and the NSF Grant PHY-1104683. ITS401 simulations were completed utilizing the Holland Comput- ${ }^{402}$ ing Center of the University of Nebraska. XD acknowledges ${ }_{404}^{403}$ PRACE for awarding access to TGCC/Curie under the Grant 405 2014112576.

[1] E. Esarey, C. B. Schroeder, W. P. Leemans, Rev. Mod. Phys. 81 (2009) 1229

[2] V. Malka, Phys. Plasmas 19 (2012) 055501

[3] K. Ta Phuoc, A. Rousse, M. Pittman, et al., Phys. Rev. Lett. 91 (2003) 195001

[4] H. Schwoerer, B. Liesfeld, H.-P. Schlenvoigt, et al., Phys. Rev. Lett. $96_{413}^{412}$ (2006) 014802

[5] K. Ta Phuoc, S. Corde, C. Thaury, et al., Nat. Photon. 6 (2012) 308

[6] A. Jochmann, A. Irman, M. Bussmann, et al., Phys. Rev. Lett. 111 (2013) 114803

[7] S. Chen, N. D. Powers, I. A. Ghebregziabher, et al., Phys. Rev. Lett. $110_{41}^{417}$ (2013) 155003

[8] N. D. Powers, I. A. Ghebregziabher, G. Golovin, et al., Nat. Photon. $8_{420}^{419}$ (2014) 28

[9] C. Liu, G. Golovin, S. Chen, et al., Opt. Lett. 39 (2014) 4132

10] E. Miura, S. Ishii, K. Tanaka, R. Kuroda, H. Toyokawa, Appl. Phys. Ex- 422 press 7 (2014) 046701

[11] H.-E. Tsai, X. Wang, J. M. Shaw, et al., Phys. Plasmas $22(2015) 023106_{425}^{424}$

[12] K. Khrennikov, J. Wenz, A. Buck, et al., Phys. Rev. Lett. 114 (2015) 195003

[13] S. P. D. Mangles, G. Genoud, S. Kneip, et al., Appl. Phys. Lett. 95 (2009) ${ }_{428}^{427}$ 181106

[14] A. Popp, J. Vieira, J. Osterhoff, et al., Phys. Rev. Lett. 105 (2010) $215001{ }_{430}^{429}$

[15] S. Y. Kalmykov, A. Beck, X. Davoine, E. Lefebvre, B. A. Shadwick, New J. Phys. 14 (2012) 033025

[16] S. Y. Kalmykov, X. Davoine, R. Lehe, A. F. Lifschitz, B. A. Shadwick, Phys. Plasmas 22 (2015) 056701

[17] D. Herrmann, L. Veisz, R. Tautz, et al., Opt. Lett. 34 (2009) 2459

[18] H. Fattahi, H. G. Barros, M. Gorjan, et al., Optica 1 (2014) 45

[19] S. Y. Kalmykov, X. Davoine, B. A. Shadwick, Nucl. Instrum. Methods in Phys. Res. A 740 (2014) 266

[20] C. M. S. Sears, E. Colby, R. Ischebeck, et al., Phys. Rev. ST Accel. Beams 11 (2008) 061301

[21] Z. Zhang, Y. Ding, A. Martinelli, Z. Huang, Phys. Rev. ST Accel. Beams 18 (2015) 030702

[22] A. Cianchi, D. Alesini, M. P. Anania, et al., Phys. Rev. ST Accel. Beams 18 (2015) 082804

[23] Z. Zhang, Y. Ding, P. Emma, et al., Phys. Rev. ST Accel. Beams 18 (2015) 090701

[24] A. Oguchi, A. Zhidkov, K. Takano, et al., Phys. Plasmas 15 (2008) 043102

[25] A. Zhidkov, J. Koga, T. Hosokai, et al., Phys. Plasmas 17 (2010) 083101
[26] N. A. M. Hafz, S. K. Lee, T. M. Jeong, J. Lee Nucl. Instrum. Methods in Phys. Res. A 637 (2011) S51

[27] P. A. Walker, N. Bourgeois, W. Rittershofer, et al., New J. Phys. 15 (2013) 045024

[28] G. G. Manahan, E. Brunetti, C. Aniculaesei, et al., New J. Phys. 16 (2014) 103006

[29] O. Lundh, C. Rechatin, J. Lim, V. Malka, J. Faure, Phys. Rev. Lett. 110 (2013) 065005

[30] S. Y. Kalmykov, B. A. Shadwick, I. A. Ghebregziabher, et al., in: Proceedings of the 2014 IEEE 41st International Conference on Plasma Sciences held with 2014 IEEE International Conference on High-Power Particle Beams (ICOPS/BEAMS 2014), Washington, DC, USA, pp. 1-6; DOI: 10.1109/PLASMA.2014.7012740

[31] W. Lu, M. Tzoufras, C. Joshi, et al., Phys. Rev. ST Accel. Beams 10 (2007) 061301

[32] N. A. Bobrova, P. V. Sasorov, C. Benedetti, et al., Phys. Plasmas 20 (2013) 020703

[33] K. Nakamura, B. Nagler, Cs. Tóth, et al., Phys. Plasmas 14 (2007) 056708

[34] A. J. Gonsalves, K. Nakamura, J. Daniels, et al., Phys. Plasmas 22 (2015) 056703

[35] P. Mora, T. M. Antonsen, Jr., Phys. Rev. E 53 (1996) R2068

[36] P. Mora, T. M. Antonsen, Jr., Phys. Plasmas 4 (1997) 217

[37] A. Pukhov, J. Meyer-ter-Vehn, Appl. Phys. B: Lasers Opt. 74 (2002) 355

[38] M. Tzoufras, W. Lu, F. S. Tsung, et al., Phys. Plasmas 16 (2009) 056705

[39] W. Zhu, J. P. Palastro, T. M. Antonsen, Jr., Phys. Plasmas 19 (2012) 033105

[40] W. Zhu, J. P. Palastro, T. M. Antonsen Jr., Phys. Plasmas 20 (2013) 073103

[41] A. F. Lifschitz, X. Davoine, E. Lefebvre, et al., J. Comput. Phys. 228 (2009) 1803

[42] R. Lehe, A. F. Lifschitz, C. Thaury, V. Malka, X. Davoine, Phys. Rev. ST Accel. Beams 16 (2013) 021301

[43] J. Faure, Y. Glinec, J. J. Santos, et al., Phys. Rev. Lett. 95 (2005) 205003.

[44] J. Schreiber, C. Bellei, S. P. D. Mangles, et al., Phys. Rev. Lett. 105 (2010) 235003

[45] A. Pipahl, E. A. Anashkina, M. Toncian, et al., Phys. Rev. E 87 (2013) 033104

[46] I. A. Ghebregziabher, B. A. Shadwick, D. P. Umstadter, Phys. Rev. ST Accel. Beams 16 (2013) 030705

[47] E. Esarey, S. K. Ride, and P. Sprangle, Phys. Rev. E 48 (1993) 3003

[48] S. K. Ride, E. Esarey, M. Baine, Phys. Rev. E 52 (1995) 5425

[49] Y. Y. Lau, Fei He, D. P. Umstadter, R. Kowalczyk, Phys. Plasmas 10 (2003) 2155

[50] C. Maroli, V. Petrillo, P. Tomassini, L. Serafini, Phys. Rev. ST Accel. Beams 16 (2013) 030706

[51] J. D. Jackson, Classical Electrodynamics, 3rd edition, Wiley \& Sons, Inc., 1998, ISBN: 978-0-471-30932-1, p. 676.

[52] S. G. Rykovanov, C. G. R. Geddes, J.-L. Vay, et al., J. Phys. B: At. Mol. Opt. Phys. 47 (2014) 234013

[53] S. M. Wiggins, R. C. Issac, G. H. Welsh, et al., Plasma Phys. Control. Fusion 52 (2010) 124032

[54] R. Weingartner, M. Fuchs, A. Popp, et al., Phys. Rev. ST Accel. Beams 14 (2011) 052801 